\title{
LA FUNCIÓN EPISTÉMICA DEL DERECHO DE LOS PUEBLOS INDÍGENAS A LA CONSULTA PREVIA EN CHILE
}

\author{
Epistemic function of the right of \\ indigenous peoples to prior consultation \\ in Chile \\ FELIPE ANDRÉs GUERRA SCHLEEF* \\ Universidad Austral de Chile \\ Gonzalo Andrés Rafael SÁnchez SANdoval** \\ Universidad de Chile
}

\begin{abstract}
RESUMEN
Este trabajo argumenta que los derechos de participación indígena constituyen mecanismos fundamentales a través de los cuales los pueblos indígenas pueden participar en la formación de la evidencia durante los procesos de toma de decisiones estatales. Sin embargo, la manera en la que se ha codificando la consulta previa indígena en Chile ha implicado invertir el principio que a este respecto opera a nivel internacional, trasladando la discusión sobre la efectividad de la ocurrencia de una afectación (lo que debiera ser el resultado de la consulta) al momento de la evaluación en concreto de su principal elemento gatillante: la susceptibilidad de afectación directa. Esto ha impedido que este derecho procedimental pueda cumplir con su doble función epistémica: habilitar la participación colectiva de los pueblos indígenas como agentes de conocimientos propios y validos, y operar como un mecanismo de visibilización de impactos para la protección de los derechos indígenas. Sin perjuicio de esto, es posible observar algunos avances jurisprudenciales recientes en que, pese a no cuestionar en términos generales el modelo regulatorio de la consulta previa indígena que se ha desarrollado en Chile, han comenzado ha enfatizar la función epistémica del derecho de los pueblos indígenas a la consulta previa.
\end{abstract}

\section{PALABRAs CLAVE}

Consulta indígena, Tribunales Ambientales, Recurso de protección de garantías constitucionales.

\section{ABSTRACT}

In this paper, it is argued that indigenous participation rights constitute fundamental mechanisms through which indigenous peoples participate in the formation of evidence during decision-making processes driven by the State. However, the way in which prior indigenous consultation has been codified in Chile has implied reversing the principle that operates at the international level in this regard, shifting the discussion on the effectiveness of the occurrence of an affectation (which should be the result of the consultation) at the time of the specific evaluation of its main trigger element: the susceptibility of direct affectation. This has prevented this procedural right from fulfilling its dual epistemic function: enabling the collective participation of indigenous peoples as agents of their own and valid knowledge, and operating as a mechanism for making visible impacts for the protection of indigenous rights. However, it is possible to observe some recent jurisprudential advances in which, despite not questioning in general terms the regulatory model of prior indigenous consultation that has been developed in Chile so far, they have begun to emphasize the epistemic function of the rights of indigenous peoples to prior consultation.

\section{KEYWORDS}

Indigenous Consultation, Environmental Courts, Remedy of Protection.

\footnotetext{
* Coordinador Área Jurídica, Observatorio Ciudadano. Profesor adjunto, Instituto de Derecho Público, Facultad de Ciencias Jurídicas y Sociales, Universidad Austral de Chile (UACh), Valdivia, Chile. Becario ANID en el Programa de Doctorado en Derecho Mención en Constitucionalismo y Derecho, Facultad de Ciencias Jurídicas y Sociales, UACh. Correo electrónico: felipe.guerra.schleef@gmail.com.

** Licenciado en Derecho, Facultad de Derecho de la Universidad de Chile (UCH). Ayudante del Centro de Derecho Ambiental de la Universidad de Chile (CDA), Santiago, Chile. Correo electrónico: sanchezsandovalgonzalo@gmail.com.
} 


\section{Introducción}

Desde la ratificación del Convenio 169 de la Organización Internacional del Trabajo (OIT) por parte de Chile en septiembre de 2008 , el foco del debate respecto a la implementación de este tratado internacional ha estado puesto en la domesticación del derecho de los pueblos indígenas a la consulta previa, ya sea por medio de su reglamentación por parte del Ejecutivo o su interpretación judicial ${ }^{1}$. En este contexto, dos de los aspectos más controversiales han sido la determinación del contenido y alcance de la "susceptibilidad de afectación directa" (SAD) como elemento que desencadena la obligación de consultar una determinada medida administrativa o legislativa, así como la noción que se ha impuesto de este derecho como uno cuyo cumplimiento se agota en la verificación de sus salvaguardas procedimentales con independencia de su resultado. De esta forma, mientras lo primero ha tenido como consecuencia acotar los casos en que se debe implementar un proceso de consulta previa, agregando exigencias que son ajenas al convenio; lo segundo ha implicado desplazar el centro para evaluar el cumplimiento (o incumplimiento) de este derecho en la conducta del propio Estado, atribuyéndole legitimidad a un resultado atendiendo solo las características del procedimiento ${ }^{2}$.

En lo que sigue, este trabajo busca examinar ambas cuestiones a partir de un análisis del tratamiento reglamentario de este derecho y la jurisprudencia reciente desarrollada por los tribunales ambientales y la Corte Suprema en el contexto de las reclamaciones ambientales y el recurso de protección. En particular nos interesa conocer hasta qué punto ha penetrado en Chile lo que denominaremos como la función epistémica del derecho de los pueblos indígenas a la consulta previa.

Argumentaremos que a nivel internacional los derechos de participación de los pueblos indígenas constituyen mecanismos fundamentales a través de los cuales estos grupos pueden participar en la formación de la evidencia durante los procesos de toma de decisiones estatales. Sin embargo, la manera en la que se ha regulado la consulta previa indígena en este país ha implicado invertir el principio que a este respecto opera a nivel internacional, trasladando la discusión sobre la efectividad de la ocurrencia de una afectación (lo que debiera ser el resultado de la consulta) al momento de la evaluación en concreto de su principal elemento gatillante: la SAD. Esto ha impedido que este derecho de carácter procedimental pueda cumplir con la doble función epistémica para la cual ha sido diseñado y que resultan fundamentales en el contexto de los procedimientos de toma de decisiones: habilitar la participación colectiva de los pueblos indígenas como agentes de conocimientos propios y validos, y operar como un mecanismo de visibilización de impactos para la protección de los derechos indígenas en contextos de diversidad cultural. Sin perjuicio de esto, es posible observar algunos avances jurisprudenciales recientes en que, pese a no cuestionar en términos generales el modelo regulatorio de la consulta previa indígena desarrollado en Chile, han comenzado ha enfatizar la función epistémica de este derecho en diversas etapas de los procesos de toma de decisiones.

Para desarrollar este argumento, en lo que sigue este trabajo se organiza en cuatro apartados. En primer lugar, se discute la forma en que la consulta previa indígena se inserta en el programa del Convenio 169, dando cuenta de las diversas narrativas que se han articulado para justificar este derecho, ya sea destacando su carácter procedimental o su función epistémica. En segundo lugar, se analiza cómo quedó recogida la consulta previa en los dos reglamentos que la regulan actualmente en Chile, poniendo especial énfasis en la forma en la que se ha codificado la SAD y la noción que se ha impuesto de este derecho como uno cuyo cumplimiento se agota en la verificación de sus salvaguardas procedimentales. En tercer lugar, se examinan los desarrollos jurisprudenciales recientes en torno a la consulta previa en el

\footnotetext{
${ }^{1}$ En este sentido, ver: DONOSO (2012), p. 174; DONOSO (2014), pp. 58-59; CORDERO (2013), pp. 73-74; LÓPEZ Y MOHR (2014), pp. 112-124; ANINAT (2014), pp. 2-3; MEZA-LOPEHANDÍA (2016), pp. 22-44; GUERRA (2017), pp. 21-26.

${ }^{2}$ MILLALEO (2014), pp. 56-57.
} 
contexto de las reclamaciones ambientales y el recurso de protección, y que permiten afirmar que un nuevo entendimiento respecto a la función epistémica de este derecho está permeando nuestro ordenamiento jurídico interno. El trabajo finaliza exponiendo las principales conclusiones.

\section{La consulta previa indígena en el Convenio 169: entre la resolución de conflictos y la visibilización de impactos}

Pese a lo natural que nos parece hoy en día ver el derecho internacional de los derechos humanos como un aliado de los pueblos indígenas, esta apuesta de los movimientos indígenas a nivel global se inscribe en un giro estratégico relativamente reciente. De hecho, desde sus orígenes teológicos hasta bien entrado el siglo XX, el derecho internacional jugó un papel central en las dramáticas historias de conquista, colonización y desposesión de los pueblos indígenas alrededor del mundo ${ }^{3}$. Esta característica genética del derecho internacional, que continuó con el establecimiento de Sociedad de las Naciones ${ }^{4}$, se reactualizó tras la Segunda Guerra Mundial, durante las décadas en las que las Naciones Unidas se ocupó de la descolonización. En este contexto, el régimen de normas de descolonización que se desarrolló en el sistema internacional eludió en gran medida los patrones indígenas de asociación y ordenamiento político que se originaron en los territorios colonizados previo a la colonización europea ${ }^{5}$. En cambio, la población de un territorio colonial como un todo integral se consideró la unidad beneficiaria de las prescripciones de descolonización, dejando los límites coloniales intactos, independientemente del carácter arbitrario de estos.

Fue en esta época en la que, en el ceno de la OIT -una agencia tripartita anterior que pasaría a las Naciones Unidas-, se redactaría el primer instrumento internacional sobre derechos indígenas: el Convenio 107 Sobre poblaciones indígenas y tribuales de 1957. Como era de esperar, este convenio reflejaba la ansiedad de los estados independientes (y recientemente independientes) sobre la amenaza de que los pueblos indígenas se valieran de las normas emergentes de autodeterminación y descolonización. Así, el Convenio 107 partía de la premisa que los principios de autogobierno y autodeterminación en relación con estos grupos podrían cumplirse a través de la asimilación y la concesión de derechos de ciudadanía plena e igualitaria a sus miembros ${ }^{6}$. Desde esta perspectiva, el programa de este tratado era simplemente hacer más inclusivo el "Estado-nación" por medio de su homogenización cultural, lo que era coherente con el liberalismo de corte individualista que inspiraba el tratamiento de las minorías en el marco internacional de derechos humanos que comenzaba a desarrollarse en ese periodo. Sin embargo, estas políticas paternalistas tuvieron como resultado una serie de consecuencias destructivas para los pueblos indígenas. En la práctica, la integración se convirtió en un concepto que significaba la extinción de las formas de vida diferentes a las de las sociedades dominantes ${ }^{7}$.

Con los pueblos indígenas asumiendo cada vez más control de la agenda internacional de derechos humanos en lo que a ellos respecta, el Convenio 107 paso rápidamente a estar en el centro de las demandas de los movimientos indígenas por reformular la respuesta internacional hacia ellos. En este contexto, en 1986 el Consejo de Administración de la OIT inició el proceso de revisión del Convenio 107, basándose, entre otras cosas, en la nueva perspectiva de una mayor autonomía para los pueblos indígenas y el reconocimiento de su control colectivo sobre sus tierras y recursos. El resultado de este proceso sería el Convenio 169, Sobre Pueblos Indígenas y Tribales en Países Independientes, adoptado por la Conferencia General de la OIT en 1989.

\footnotetext{
${ }^{3}$ EIDE (2010), pp. 34-35.

${ }^{4}$ NIEZEN (2003), pp. 31-36.

${ }^{5}$ ANAYA (2000), p. 43.

${ }^{6}$ ANAYA (1991), pp. 31-32.

${ }^{7}$ ANAYA (2000), p. 44.
} 
De esta manera, distanciándose de la filosofía de su antecesor, el Convenio 169 parte del reconocimiento de "[...] las aspiraciones de esos pueblos a asumir el control de sus propias instituciones y formas de vida y de su desarrollo económico y a mantener y fortalecer sus identidades, lenguas y religiones, dentro del marco de los Estados en que viven" (preámbulo, Convenio 169). Sobre esta premisa, el convenio incluye disposiciones que cubren una amplia variedad de temas: protección de la integridad cultural indígena, tierras y recursos, derecho a conservar sus sistemas jurídicos e instituciones propias, no discriminación en las esferas del bienestar social, entre otros. Sin embargo, como ha sido señalado por la OIT, el establecimiento de mecanismos apropiados y eficaces para la consulta y la participación de los pueblos indígenas en relación con las cuestiones que les conciernen son la "piedra angular" del Convenio $169^{8}$, al constituir disposiciones claves sobre las cuales "reposa la aplicación de las demás disposiciones" ${ }^{\prime}$.

Esto ha significado que una parte importante de la implementación del Convenio se refiera a la interpretación de sus disposiciones relativas a la participación y consulta, convirtiéndose en temas de considerable debate, tensión y arreglos regulatorios sobre políticas públicas en comparación con otras áreas, como los derechos territoriales o a conservar sus sistemas jurídicos propios, entre otros. Una razón obvia de esto es la intersección entre las normas sobre consulta y participación, y los proyectos de inversión públicos y privados. Como instrumento vinculante, los estándares desarrollados en torno al Convenio 169 han sido fundamentales en las estrategias de impugnación indígena y sus reclamos territoriales, lo que ha desencadenado debates virulentos sobre los riesgos para (más que de) los proyectos de inversión ${ }^{10}$.

Estas tensiones se observan con claridad en las narrativas en competencia que se han venido desarrollando en torno a la consulta previa. Mientras los Estados y la OIT han enfatizado en la consulta como instrumento de gobernanza para institucionalizar el diálogo, asegurar procesos de desarrollo incluyentes y prevenir y resolver conflictos ${ }^{11}$, han prestado menor atención a este derecho como mecanismo procedimental para proteger los derechos sustantivos de los pueblos indígenas (como es el caso de sus de sus derechos culturales, territoriales o a mantener sus sistemas jurídicos propios).

El énfasis puesto en la resolución de conflictos ha acentuado la funcionalidad de los derechos de participación indígena en la armonización intereses contrapuestos. Este enfoque, sumado a la aversión al "veto" que se ha instalado en el contexto institucional de la $\mathrm{OIT}^{12}$, en muchos caso se ha traducido en la exigencia de una amplia flexibilidad a los pueblos indígenas respecto a sus posiciones, intereses y derechos, ocultando la intransigencia de sus interlocutores, tanto estatales como empresariales. Asimismo, esto ha llevado a centrar la atención exclusivamente en el cumplimiento de las garantías procedimentales que rigen estos mecanismos de dialogo -dando lugar a lo que Millaleo identifica como una versión rawlsiana de "justicia puramente procedimental" ${ }^{13}$-, eludiéndose las discusiones de fondo respecto a los impactos que una determinada medida puede tener para un pueblo o comunidad, o si la decisión que en definitiva se adopte respeta los demás derechos garantizados por el Convenio 169.

En efecto, además de ser un instrumento para la prevención y resolución de conflictos, la consulta previa busca habilitar la participación colectiva de los pueblos indígenas desde su especificidad, como un medio para la protección de sus derechos colectivos en contextos de

\footnotetext{
${ }^{8}$ COMISIÓN DE EXPERTOS EN APLICACIÓN DE CONVENIOS Y RECOMENDACIONES (2009), p. 7.

${ }^{9}$ COMISIÓN DE EXPERTOS EN APLICACIÓN DE CONVENIOS Y RECOMENDACIONES (2006), párr. 6.

10 LARSEN (2019) pp. 4-5.

${ }^{11}$ En este sentido, por ejemplo, los pronunciamiento de la CEACR de la OIT en sus observaciones a Estados: Guatemala (2005 y 2018); Bolivia (2005); Perú (2005 y 2009). Asimismo, las observaciones generales de la CEACR sobre los derechos de consulta y participación, CEACR (2009). En el mismo sentido, se han pronunciado los comités tripartitos instaurados para examinar reclamaciones en contra de México (2006, GB.296/5/3, párr. 44) y Brasil (2009, GB.304/14/7, párr. 42), entre otros. En: OFICINA INTERNACIONAL DEL TRABAJO (2019).

12 COMISIÓN DE EXPERTOS EN APLICACIÓN DE CONVENIOS Y RECOMENDACIONES (2011), p. 11.

${ }^{13}$ MILLALEO (2014), pp. 56-57.
} 
diversidad cultural, lo que requieren de espacios especiales de diálogo para hacer visibles impactos que podrían no ser transparentes para la cultura dominante ${ }^{14}$. Esto, se traduce en que los Estados tienen la obligación de ajustar o incluso cancelar una determinada decisión con base en los resultados de la consulta con los pueblos indígenas ${ }^{15}$. Desde esta perspectiva, el hecho de que un proceso de consulta concluya sin acuerdo o consentimiento de los pueblos indígenas, no habilita al Estado para adoptar decisiones que vulneren los otros derechos de carácter sustantivo reconocidos por el Convenio 169. Esto, por supuesto, es sin perjuicio de los casos en que el derecho internacional requiere expresamente del consentimiento indígena como un requisito para adoptar una determinada decisión estatal ${ }^{16}$.

Desde esta perspectiva, la consulta indígena se erige como un dispositivo procedimental cuyo objetivo es remediar (hasta cierto punto) un tipo particular de "injusticia epistémica" ${ }^{17}$, que ocurre cuando determinados sujetos sujetos o grupos son excluidos de participar como agentes de conocimiento potenciales en las prácticas de indagación epistémica, por su pertenencia a una o más identidades sociales marginadas ("marginación hermenéutica"). Así, los derechos de participación indígena pueden ser comprendidos como mecanismos que buscan corregir lo que Schmidt denomina como "fallas de reconocimiento" y "fallas de acceso" ${ }^{18}$ en las prácticas cognoscitivas que, en este caso, anulan la capacidad de los pueblos indígenas de incidir como participantes epistémicos en la generación de conocimientos vinculados a los procesos de toma de decisiones estatales que los podrían afectar.

La dimensión epistémica de la consulta previa es, además, consistente con el principal elemento que gatilla este deber estatal contenido en el artículo 6 del Convenio 169: la SAD ${ }^{19}$. En efecto, de acuerdo con el convenio, para que se desencadene un proceso de consulta, por regla general, se requiere que la medida que se pretende adoptar pueda afectar los intereses y derechos de algún grupo indígena ${ }^{20}$. No es necesario que exista certeza de que se producirá una afectación, ya que, como se señaló, es justamente la opacidad de las valoraciones ajenas en un contexto de diversidad cultural lo que hace necesaria la incorporación de mecanismos especiales de comunicación intercultural ${ }^{21}$.

Asimismo, esto es consistente con el objetivo que el Convenio 169 le atribuye a la consulta previa indígena en el contexto del desarrollo de actividades extractivas en los territorios de los pueblos indígenas: "[...] determinar si los intereses de esos pueblos serían perjudicados y en qué medida antes de emprender o autorizar cualquier programa de prospección o explotación de los recursos existentes en sus tierras" (art. 15.2) ${ }^{22}$. De esta manera, la consulta previa indígena cumple una función epistémica clave en los procesos de toma de decisiones, al apoyar la generación de evidencia respecto a los impactos que una determinada medida puede tener sobre los pueblos indígenas, con el objetivo de que la decisión final que se adopte se acomode a las perspectivas, intereses y derechos sustantivos de estos grupos, históricamente marginados de las instancias decisorias al interior de los Estados de colonos que los han subordinado.

\footnotetext{
${ }^{14}$ En este sentido: COMISIÓN INTERAMERICANA DE DERECHOS HUMANOS (2015), párr. 176; MEZA-LOPEHANDÍA (2016), p. 22.

${ }^{15}$ COMISIÓN INTERAMERICANA DE DERECHOS HUMANOS (2009), párrs. 323-325.

${ }^{16}$ Para un análisis general de los casos en que el derecho internacional requiere expresamente del consentimiento indígena, ver: COMISIÓN INTERAMERICANA DE DERECHOS HUMANOS (2015), párrs. 183-193.

${ }^{17}$ Este concepto fue articulado analíticamente por primera vez por Miranda Fricker. En este sentido, ver: FRICKER (2007), pp. 237279.

${ }^{18}$ Ver: SCHMIDT (2019), pp. 58-62.

${ }^{19}$ De acuerdo con el artículo 6 № 1, literal a) del Convenio 169: “1. Al aplicar las disposiciones del presente Convenio, los gobiernos deberán: [...] a) consultar a los pueblos interesados, mediante procedimientos apropiados y en particular a través de sus instituciones representativas, cada vez que se prevean medidas legislativas o administrativas susceptibles de afectarles directamente".

${ }^{20}$ Ver: CONSEJO DE DERECHOS HUMANOS DE LAS NACIONES UNIDAS (2009), párr. 43.

${ }^{21}$ En este sentido: AYLWIN et al. (2013), p. 397; MEZA-LOPEHANDÍA (2016), p. 37.

22 Esta es la razón por la cual en reiteradas oportunidades los organismos de control de la OIT han señalado que los "[...] riesgos [ambientales de las actividades extractivas] deben ser objeto de la consulta prevista en el artículo 15, 2) del Convenio en conexión con los estudios [de impacto socioambiental] contemplados en el artículo 7, 3, del Convenio". Observación de la CEACR a Guatemala en 2005, entre otras. En: OFICINA INTERNACIONAL DEL TRABAJO (2019), p. 25.
} 


\section{Las políticas de implementación de la consulta indígena en Chile}

La discusión previa a la aprobación del Convenio 169 se redujo a las cuestiones planteadas por el Tribunal Constitucional en el año 2000, al resolver el requerimiento de inconstitucionalidad presentado por un grupo de parlamentarios de oposición al gobierno de la época. Si bien el Tribunal Constitucional rechazó la acción, limitó severamente el contenido de este tratado, al señalar que solo sus disposiciones sobre consulta y participación -contenidas en sus artículos 6 y 7 , respectivamente- eran autoejecutables (self-executing), por tener el contenido y precisión necesarias que las habilitaban para ser aplicadas sin otro tramite como fuentes de derecho interno ${ }^{23}$.

Tras la promulgación del Convenio 169, y antes de que entrará en vigor, el gobierno adoptó como estrategia la regulación del derecho de consulta indígena, a través de la dictación del Decreto Supremo 124/2009 del Ministerio de Planificación, cuyo objetivo era reglamentar del artículo 34 de la denominada Ley Indígena (Ley № 19.253 de 1993). Aunque el ejecutivo reconoció que este reglamento tenia un carácter transitorio mientras se definía con los pueblos indígenas un modelo de consulta permanente, su texto reflejaba lo contrario. Esto apreciaba, entre otras cosas, en el carácter genérico de sus disposiciones y el intento deliberado de sustraer de la consulta a los proyectos de inversión, cuestión que sería férreamente defendida por el Ejecutivo a medida que las organizaciones indígenas comenzaron a exigir la implementación de este derecho a través del recurso de protección de garantías constitucionales.

Si bien las decisiones de la Corte Suprema fueron inicialmente favorables a la posición del gobierno, luego de algunas oscilaciones esto cambiaría, lo que despertó críticas desde sectores empresariales que comenzaron a pedir una regulación más detallada para eliminar las zonas grises que permitan una "judicialización" percibida. En respuesta a estas presiones, se desencadenarían nuevas acciones regulatorias en los campos de la evaluación de impacto ambiental y la consulta indígena. Esto daría lugar a los dos reglamentos que regulan actualmente la consulta indígena: el Decreto Supremo 40/2013 del Ministerio del Medio Ambiente, que aprobó el nuevo reglamento del Sistema de Evaluación de Impacto Ambiental (SEIA); y el Decreto Supremo 66/2014 del Ministerio de Desarrollo Social, que estableció el procedimiento general de consulta indígena y derogó el Decreto Supremo 124/2009.

Si bien estas reglamentaciones han sido objeto de múltiples cuestionamientos tanto por razones de forma como de fondo, por caer por debajo de los estándares del Convenio $169^{24}$, sus disposiciones reflejan delicados actos de equilibrio para actuar de conformidad con y promover una comprensión minimalista del Convenio $169^{25}$. A continuación se examinará cómo ha quedado recogida la consulta previa en estos dos reglamentos, poniendo especial énfasis en la forma en la que se ha codificado la exigencia de una afectación como elemento gatillante de la consulta y la noción que se ha impuesto de este derecho como uno cuyo cumplimiento se agota en la verificación de sus salvaguardas procedimentales con independencia de su resultado.

\subsection{La reglamentación de la consulta en el SEIA}

El ensamblaje de la consulta previa indígena en el SEIA no ha sido una tarea fácil, ya que ha estado mediada por la estructura institucional y las políticas del conocimiento que operan en este procedimiento administrativo ${ }^{26}$. Particularmente importante en este contexto ha sido la organización en dos niveles de la evaluación ambiental de proyectos. De esta forma, el

\footnotetext{
${ }^{23}$ Tribunal Constitucional, Rol № 309-2000, de 4 de agosto de 2000.

${ }^{24}$ En este sentido: INSTITUTO NACIONAL DE DERECHOS HUMANOS (2013), pp. 18-31; MILLALEO (2014), pp. 60-97; FUNDACIÓN PARA EL DEBIDO PROCESO (2015); COMISIÓN INTERAMERICANA DE DERECHOS HUMANOS (2015), pp. 25-27 y pp. $45-47$.

${ }^{25}$ LARSEN (2016), p. 8.

${ }^{26}$ En este sentido, ver: KELLY (2019), pp. 232-233.
} 
titular de un proyecto "susceptible de causar impacto ambiental" 27 , que debe someterse obligatoriamente al SEIA previo a su ejecución, lo hace, por regla general, presentando una Declaración de Impacto Ambiental (DIA), salvo que pueda generar o presentar alguno de los efectos, características o circunstancias señalados en los literales del artículo 11 de la Ley $\mathrm{N}^{\circ}$ 19.300 ("impactos significativos") ${ }^{28}$, en cuyo caso debe presentar un Estudio de Impacto Ambiental (EIA) y proponer medidas para hacerse cargo de dichos efectos ambientales adversos. En ambos casos la evidencia es preparada por los proponentes, quienes, junto con demostrar que sus iniciativas se ajustan a la normativa ambiental, tienen la carga de probar que sus proyectos no genera impactos significativos (caso de la DIA ${ }^{29} \mathrm{o}$, si los genera, que se hace cargo adecuadamente de estos proponiendo medidas de mitigación, reparación y/o compensación adecuadas (caso del EIA) ${ }^{30}$. Dicha evidencia es ponderada por los organismos sectoriales con competencias ambientales que participan de la evaluación, quienes pueden hacer observaciones y requerir aclaraciones o ampliaciones, siendo finalmente el proyecto calificado, por regla general, por una comisión donde concurren a votar exclusivamente autoridades políticas. De esta manera, el procedimiento administrativo de evaluación ambiental puede ser concebido como un procedimiento probatorio con una doble finalidad epistémica, ya que, por una parte, busca establecer si determinadas actividades se ajustan (o no) a la normativa ambiental aplicable $y$, por la otra, determinar preventivamente el impacto (riesgo) que estas actividades pueden tener en el medio ambiental.

Luego de la entrada en vigencia del Convenio 169 (septiembre de 2009), el gobierno continuó operando como antes, negando la procedencia de la consulta indígena en el contexto de la evaluación ambiental de proyectos de inversión y señalando que los mecanismos de participación ciudadana en el SEIA (en aquella época restringidos exclusivamente a los EIA) permitían dar por cumplida esta obligación. Esto provocó varios conflictos con comunidades indígenas, las cuales empezaron a canalizar sus reclamos por la vía del recurso de protección, principal remedio judicial disponible en aquella época para impugnar autorizaciones ambientales inconsultas ${ }^{31}$.

Aun cuando las decisiones iniciales de la Corte Suprema fueron favorables a la posición del gobierno, tras algunas oscilaciones, comenzó a afirmar que la consulta previa del convenio era algo diferente a la participación ciudadana incluida en la Ley № 19.300, constituyendo una realización del derecho constitucional a la participación igualitaria ${ }^{32}$. Sin embargo, al hacer esto la Corte homologó la afectación exigida para gatillar un proceso de consulta indígena (la SAD) con los "impactos significativos" que dan origen a la obligación de presentar un EIA, y ordenó que los mecanismos de participación ciudadana se debían ajustar a los estándares del Convenio $169^{33}$. De esta manera, aun cuando la Corte identificó la diferencia fundamental entre la participación ciudadana y la consulta indígena, introdujo una exigencia de magnitud a la afectación exigida por el Convenio para gatillar este derecho que es ajena al estándar internacional, exceptuando de dicha obligación a todos aquellos proyectos que ingresan al SEIA por medio de simples DIA ${ }^{34}$.

Con todo, en el plano hermenéutico la referida homologación pareciera ser un problema más aparente que real. En efecto, si uno de los "impactos significativos" cuya concurrencia hace exigible que un proyecto se evalúe por medio de un EIA es la localización próxima a población protegida susceptible de ser afectada (art. 11 letra d) de la Ley $N^{\circ} 19.300$ ), forzoso

\footnotetext{
${ }^{27} \mathrm{El}$ art. 10 de la Ley № 19.300, de 1994 contiene una tipología taxativa de 20 proyectos o actividades que se consideran "susceptibles de causar impacto ambiental, en cualquiera de sus fases", y que, por tanto, deben someterse de forma obligatoria al SEIA previo a su ejecución.

${ }^{28}$ Si bien la Ley № 19.300, de 1994 no denomina a los efectos, características o circunstancias señaladas en su artículo 11 como

“impactos significativos", sí lo hace el Decreto Supremo № 40/2013, del Ministerio del Medio Ambiente (art. 2 literal e).

${ }^{29}$ Art. 19, inciso 3o, de la Ley № 19.300, de 1994.

${ }^{30}$ Art. 16, inciso final, de la Ley № 19.300, de 1994.

${ }^{31}$ CORDERO (2013), pp. 73-74.

32 MEZA-LOPEHANDÍA (2016), p. 33; GUERRA (2017), pp. 22-24; MEZA-LOPEHANDÍA (2018), p. 131.

${ }^{33}$ DONOSO (2014), pp. 61-62; CORDERO (2013), pp. 75-78; ANINAT (2014), p. 4; LÓPEZ Y MORH (2014), pp. $120-124$.

${ }^{34}$ ANAYA (2012), p. 14; COMISIÓN INTERAMERICANA DE DERECHOS HUMANOS (2015), p. 92; MEZA-LOPEHANDÍA (2016), p. 30; GUERRA (2017), pp. 22-24
} 
es concluir que la $S A D$ es en sí misma un impacto significativo. De ahí que, como se desarrollará a continuación, la magnitud y dimensión de la afectación (en términos de significancia) para gatillar un proceso de consulta indígena en el SEIA es un requisito que se ha impuesto por instrumentos infra legales, pero no encuentra justificación en el artículo 11 letra d) de la Ley № 19.300 y el Convenio 169.

Dado que la ratificación del Convenio 169 coincidió con la reforma a la institucionalidad ambiental -Ley № 20.417 de 2010, que "Crea el Ministerio del Medio Ambiente, el Servicio de Evaluación Ambiental y la Superintendencia del Medio Ambiente"-, el 12 de agosto de 2013 se pública el Decreto Supremo № 40/2013, del Ministerio del Medio Ambiente, que aprobó una nueva reglamentación para el SEIA (en adelante el "Reglamento del SEIA"), incorporando disposiciones específicas sobre la consulta indígena en el contexto de las evaluaciones ambientales de proyectos. A pesar de que dicha reforma implicó ampliar los mecanismos de participación de la ciudadanía a las DIA, la consulta indígena continuó limitada exclusivamente a los EIA, consolidándose el modelo restrictivo desarrollado jurisprudencialmente por la Corte, al cual se le han adicionado nuevas limitaciones ${ }^{35}$.

En efecto, el artículo 85 del Reglamento del SEIA señala que, en el caso de que un proyecto o actividad genere o presente alguno de los impactos significativos indicados en los artículos 7, 8 y 10-reasentamiento de comunidades humanas, o alteración significativa de los sistemas de vida y costumbres de grupos humanos; Localización próxima a población protegida; y Alteración del patrimonio cultural-, en la medida que se afecte directamente a uno o más grupos humanos pertenecientes a pueblos indígenas, el Servicio de Evaluación Ambiental (SEA) deberá diseñar y desarrollar un proceso de consulta de buena fe, que contemple mecanismos apropiados según las características socioculturales propias de cada pueblo y a través de sus instituciones representativas, de modo que puedan participar de manera informada y tengan la posibilidad de influir durante el proceso de evaluación ambiental. A su vez, el inciso 20 del artículo 85 del Reglamento del SEIA indica que en el proceso de consulta "[...] participarán los pueblos indígenas afectados de manera exclusiva y deberá efectuarse con la finalidad de llegar a un acuerdo o lograr el consentimiento. No obstante, el no alcanzar dicha finalidad no implica la afectación del derecho a la consulta".

La homologación que se ha hecho entre la SAD y los "impactos significativos" en el SEIA y la incorporación de criterios de extensión y magnitud de la intervención para evaluar la circunstancia del literal d) de la Ley $\mathrm{N}^{\circ} 19.300$, ha supuesto elevar el grado de certeza requerido para desencadenar una consulta indígena en un caso concreto, ya sea exigiendo que se acredite que "se genera o presenta" efectivamente un reasentamiento de comunidades humanas, una alteración significativa de los sistemas de vida y costumbres de grupos humanos o una alteración del patrimonio cultural, o bien que exista una "duda razonable, cierta y verosímil" de que se producirá ${ }^{36}$. Esto ha trasladado la discusión sobre la efectividad de la ocurrencia o no de una afectación (lo que debiera ser el resultado de la consulta) al momento de la evaluación en concreto de la procedencia de la consulta, anulando su función epistémica en este contexto institucional: habilitar la participación de los pueblos indígenas en la incorporación de elementos de juicio durante el proceso de formación de la prueba en el procedimiento de evaluación de impacto ambiental.

Dado que la procedencia de la consulta indígena en el SEIA está directamente vinculada con la generación de impactos significativos, el SEA ha entendido que su objeto se reduce únicamente a "[...] obtener acuerdos con respecto a la pertinencia de las medidas de mitigación, reparación o compensación del proyecto en relación a los impactos significativos sobre los [...] [grupos indígenas], y otras acciones ambientales del proyecto para resguardar a [...] [estos grupos] de potenciales afectaciones" ${ }^{37}$. Esto se ha traducido en la práctica en que las

\footnotetext{
${ }^{35}$ GUERRA (2017), pp. 24-25.

${ }^{36}$ SEA (2016), pp. 12-22.

37 SEA (2016), p. 31.
} 
consultas en el SEIA operen únicamente como un dispositivo de negociación de medidas, en lugar de mecanismos que contribuyen a la conformación de evidencia.

Lo anterior se complejiza si se considera cómo se inserta la acreditación (o descarte) de la SAD en el diseño institucional del procedimiento indagatorio de evaluación ambiental, que radica esta cuestión, primariamente en el titular ${ }^{38}$, lo que genera incentivos para que los proponentes subestimen y minimicen los impactos que sus iniciativas tendrán sobre los territorios de los pueblos indígenas. Para evitar este problema, el nuevo Reglamento del SEIA estableció en su artículo 86 la obligación del SEA de reunirse con los grupos indígenas localizados en el área en que se desarrollará el proyecto, cuando ingresa a evaluación ambiental desconociendo impactos significativos respecto de ellos, pero se emplaza en tierras indígenas, áreas de desarrollo indígena o en sus cercanías. El objetivo de esta obligación es que la autoridad ambiental pueda constatar en terreno si los impactos sobre los grupos indígenas han sido abordados adecuadamente por el proponente y, en caso de no ser así, poner término anticipado a la evaluación por falta de información relevante o esencial, o por haberse ingresado el proyecto a través de un incorrecto instrumento de evaluación ${ }^{39}$.

Pese a los términos imperativos en que está redactado el artículo 86 del Reglamento del SEIA, el SEA lo ha interpretado como una norma facultativa ${ }^{40}$, lo que ha significado que sea escasamente aplicada en la práctica. Además, cuando el SEA ha realizado reuniones amparándose en este mecanismo pre-consultivo, por lo general, han sido meramente informativas y con miembros individuales de los grupos indígenas afectados, sin garantizar su participación colectiva. Esto ha llevado a una pérdida sustancial de autonomía para estos grupos, ya que la determinación final de su afectación en el SEIA acaba siendo definida por un tercero (el titular del proyecto) y el SEA, apoyados por consultores contratados por el primero, sin la participación y control de las comunidades afectadas ${ }^{41}$. De esta manera, se ha incapacitado a los pueblos indígenas como agentes epistémicos calificados para participar en la definición de su propia afectación, reproduciéndose paradójicamente las prácticas asimilacionistas y coloniales que la introducción de los derechos de participación indígena busca remediar.

Estas restricciones se expresan en el reducido número de procesos de consulta indígena que han sido implementados hasta el momento en el SEIA. Según cifras del SEA ${ }^{42}$, desde la entrada en vigencia del Convenio 169 (septiembre de 2009) hasta octubre de 2020, habían ingresado al SEIA un total de 11.917 proyectos, que en conjunto sumaban poco más de USD \$ 550.760 millones. De estos proyectos, 541 ingresaron como EIA, mientras que el resto (11.376) lo hicieron como simples DIA. A su vez, del total de proyectos ingresados como EIA, tan solo 55 han sido sometidos a un proceso de consulta indígena en los más de diez años de vigencia del Convenio 169 en Chile, de los cuales 47 se encontraban finalizados y 8 estaban en proceso. Por otra parte, en lo que dice relación con el estado de los proyectos sometidos a consulta en el SEIA, 35 iniciativas habían sido aprobadas, 9 se encontraban en evaluación, y solo 4 habían sido rechazadas, mientras que 7 fueron desistidas por los propios titulares.

\subsection{El Reglamento general de consulta}

El 4 de marzo de 2014 se publica el Decreto Supremo № 66/2013 del Ministerio de Desarrollo Social, por medio del cual se derogó el Decreto Supremo № 124/2009 del Ministerio de Planificación y se estableció un procedimiento administrativo especial de aplicación general para la consulta previa en virtud del Convenio 169 (en adelante el "Reglamento general de

\footnotetext{
${ }^{38}$ SEA (2016), pp. 21-22.

${ }^{39}$ GOBIERNO DE CHILE (2012), p. 16.

${ }^{40}$ En este sentido, ver: SEA (2016), pp. 16-17 y 21-22.

${ }^{41}$ MEZA-LOPEHANDÍA (2016), p. 37.

42 Reporte sobre los proyectos sometidos al SEIA con proceso de consulta indígenas, disponible en: https://seia.sea.gob.cl/pci/proyectos_en_pci.php.
} 
consulta"). Al igual que la reglamentación de la consulta en el SEIA, el artículo 3 del Reglamento general de consulta, al referirse al "Cumplimiento del deber de Consulta", señala que:

"El órgano responsable deberá realizar los esfuerzos necesarios para alcanzar el acuerdo o el consentimiento de los pueblos afectados, dando cumplimiento a los principios de la consulta a través del procedimiento establecido en este reglamento. Bajo estas condiciones, se tendrá por cumplido el deber de consulta, aun cuando no resulte posible alcanzar dicho objetivo".

Bajo esta interpretación, la falta de acuerdo o, en su caso, el abandono de las comunidades del proceso consultivo, "[...] no resta validez al procedimiento a condición que se hayan cumplido con todos los requerimientos procedimentales para convocar a una consulta previa, libre, informada, culturalmente apropiada y de buena fe" ${ }^{\prime 3}$. Esto implica que el foco para evaluar el cumplimiento o incumplimiento de la obligación se pone en la conducta del Estado $^{44}$.

Por otra parte, el Reglamento general de consulta define en su artículo 7 la SAD, indicando tanto para el caso de las medidas administrativas o legislativas, que esta se producirá cuando "[...] sean causa directa de un impacto significativo y específico sobre los pueblos indígenas en su calidad de tales, afectando el ejercicio de sus tradiciones y costumbres ancestrales, prácticas religiosas, culturales o espirituales, o la relación con sus tierras indígenas" (incisos 2ㅇ y 3 o). El concepto de SAD introducido por el Reglamento general de consulta fue una de las cuestiones más conflictivas en el proceso de consulta indígena que tuvo lugar durante su elaboración y la razón por la cual muchas organizaciones representativas de pueblos indígenas se restaron de dicho proceso.

La exigencia de que la afectación sea "significativa" fue incluida para diferenciar entre medidas de menor y mayor impacto. Sin embargo, este reglamento no entrega ningún criterio respecto a qué determina la significancia de un impacto, cuestión que no ocurre en el SEIA, donde el reglamento de este instrumento de gestión ambiental desarrolla con más detalle esta cuestión ${ }^{45}$. Además de que esta distinción es ajena al Convenio 169 y no está respaldada por la práctica de los países ratificantes, ha implicado dejar en manos del Estado la determinación unilateral de si en un caso en concreto se gatilla la consulta. Para lo anterior, el órgano responsable puede solicitar un informe de procedencia a la Subsecretaría de Servicios Sociales del Ministerio de Desarrollo Social (artículo 13, Reglamento general de consulta), organismo que ha desarrollado una práctica administrativa contraria a la consulta, convirtiendo este mecanismo de diálogo intercultural en una verdadera excepción en los hechos.

\section{La recepción de la dimensión epistémica de la consulta en la jurisprudencia}

Tras la adopción de los reglamentos examinados previamente, las discusiones en torno a la consulta previa en los tribunales de justicia tendieron a inmovilizarse, aplicándose dichas regulaciones sin mayores cuestionamientos en las controversias que se suscitaban entre organizaciones indígenas, órganos del Estado y actores empresariales. Sin embargo, en el último tiempo este agotamiento de las discusiones sobre la implementación del derecho de los pueblos indígenas a la consulta previa por los tribunales pareciera estar cambiando, lo que se evidencia principalmente en la manera en la que ha estado permeando a la jurisprudencia lo que aquí hemos denominado como la dimensión epistémica de la consulta. En lo que sigue se analizaran las decisiones judiciales que despliegan este nuevo entendimiento tanto en el contexto de la institucionalidad ambiental como de la regulación general del derecho de los pueblos indígenas a la consulta previa en Chile.

\footnotetext{
${ }^{43}$ NÚÑ̃EZ (2015), p. 34

${ }^{44}$ CARMONA (2020), p. 349.

${ }^{45}$ MILLALEO (2014), p. 85.
} 


\subsection{La dimensión epistémica de la consulta previa en la institucionalidad ambiental: las reuniones previas del artículo 86 del Reglamento del SEIA y la causal de ingreso del artículo 11 d) de la Ley № 19.300}

Como parte de la reforma de la institucionalidad ambiental, en junio de 2012 se dictó la Ley № 20.600, que creó tres tribunales ambientales distribuidos territorialmente en tres macrozonas en el país. Esto abrió -no sin problemas de coordinación que se mantienen hasta nuestros días- un nuevo foro especializado para la adjudicación de conflictos relativos a la implementación de los derechos de los pueblos indígenas en el contexto de la evaluación ambiental de proyectos de inversión ${ }^{46}$.

$\mathrm{Si}$ bien estos nuevos órganos jurisdiccionales especializados han aplicado sin cuestionamiento el estrecho marco regulatorio diseñado para implementar la consulta indígena en el SEIA ${ }^{47}$, la dimensión epistémica de la consulta indígena ha comenzado a permear en recientes sentencias de los Tribunales Ambientales. Así se aprecia, por un lado, en los debates que están teniendo lugar ante el Tercer Tribunal Ambiental en relación con la implementación del artículo 86 del Reglamento del SEIA y, por el otro, en la reciente sentencia del Primer Tribunal Ambiental que interpreta la legislación ambiental a la luz de los estándares del Convenio 169.

La primera vez que un tribunal ambiental se pronunció sobre la implementación de las reuniones previas del artículo 86 del Reglamento del SEIA, fue en el caso de la DIA del proyecto "Ampliación Minicentral Hidroeléctrica Las Flores", resuelto por el Tercer Tribunal Ambiental ${ }^{48}$. Si bien una de las principales controversias en este caso decía relación con el fraccionamiento del proyecto para evitar la evaluación ambiental de la integridad de sus partes y obras, las tres comunidades mapuche reclamantes también cuestionaron que el SEA había descartado ilegalmente la SAD respecto de ellas porque no había implementado correctamente las reuniones previas reguladas en el artículo 86 del Reglamento del SEIA. Esto se debía, principalmente, a que la autoridad ambiental solo había sostenido reuniones individuales con ciertos miembros de una de las comunidades mapuche próximas y no se conocía que tipo de información les había suministrado para efectos de que de que proporcionaran una opinión fundada.

Junto con acoger la reclamación de las comunidades por haberse comprobado el fraccionamiento del proyecto, el Tribunal Ambiental señaló que el art. 86 impone al SEA un deber de "verificación", cuyo objetivo es "[...] contrarrestar la información proporcionada en la DIA con la que se pueda levantar con las reuniones en terreno con los grupos humanos indígenas" ${ }^{\prime 9}$. Para esto, resultaba clave que este organismo adoptara:

\footnotetext{
“[...] un rol activo en la generación de esas reuniones y en la recopilación de la información, siendo un estándar mínimo exigible que se reuniera con los grupos representativos del área de influencia, y si ello no fuere posible, al menos que se justifique adecuadamente esa imposibilidad en el procedimiento administrativo" 50 .
}

De esta forma, a juicio de este tribunal, estas reuniones constituyen un "trámite esencial" en el procedimiento de evaluación ambiental, al ser "[...] una norma especial para los proyectos que se emplazan en las cercanías de pueblos indígenas, inspirada en el principio colaborativo, preventivo y en la protección de los grupos humanos sensibles que puedan verse afectados por el proyecto" ${ }^{51}$. Así las cosas:

\footnotetext{
46 OBSERVATORIO CIUDADANO (2018), pp. 18-19.

${ }^{47}$ En este sentido ver: GUERRA (2017).

${ }^{48}$ Tercer Tribunal Ambiental, Rol № R-78-2018, de 7 de junio de 2019.

49 Tercer Tribunal Ambiental, Rol № R-78-2018, de 7 de junio de 2019, considerando 59으.

50 Tercer Tribunal Ambiental, Rol № R-78-2018, de 7 de junio de 2019, considerando 59․

51 Tercer Tribunal Ambiental, Rol № R-78-2018, de 7 de junio de 2019, considerando 67ㅇ.
} 
"[...] a diferencia de los demás proyectos o actividades, cuando éstos se ubican en las cercanías de población protegida, la potestad de poner término anticipado, de acuerdo al art. 48 del Reglamento del SEIA, no solo debe basarse en los informes sectoriales de los órganos con competencia ambiental, sino además en la información que se recabe conforme al art. 86 inciso $2^{\circ}$ del Reglamento del SEIA. De esta manera, si no se da cumplimiento adecuado a esta obligación, entonces la decisión de continuar con la evaluación ambiental resulta ilegal o arbitraria, por haberse ejercido la potestad en una forma diferente a la establecida en el Reglamento, habiéndose prescindido de la más fiable de las informaciones, esto es, la recopilada en terreno con los grupos humanos indígenas" 52 .

Como es posible apreciar, la interpretación que realiza este tribunal ambiental de las reuniones del art. 86 enfatiza la doble función epistémica que estas debieran desempeñar en los procedimientos de evaluación: habilitar la participación colectiva de los pueblos indígenas como agentes de conocimiento desde sus especificidades y operar como un mecanismo de visibilización de impactos para la protección de los derechos indígenas en contextos de diversidad cultural. Sin embargo, luego de este importante precedente el Tercer Tribunal Ambiental literalmente abandonó esta línea argumental para dar paso a una comprensión más formalista de estas reuniones en el contexto de los procedimientos de evaluación ambiental. Así se aprecia en las sentencias de este tribunal ambiental en los casos de los proyectos "Piscicultura de Re-circulación Lago Balmaceda" 53 , "Piscicultura San Joaquín" 54 "Parque Eólicos Las Viñas" ${ }^{55}$ y "Parque Eólico Vergara"56. Mientras en el primero de estos casos el Tercer Tribunal Ambiental eludió el debate por razones formales (supuesta falta de congruencia entre la reclamación administrativa y la judicial) ${ }^{57}$, y en el segundo por acoger la reclamación respecto de otra alegación, en el caso de los parques eólicos abandonó derechamente su jurisprudencia previa, desconociendo el carácter esencial de estas reuniones cuando su omisión "[...] no ha tenido influencia en la decisión terminal" 58.

Sin embargo, al resolver el recurso de casación presentado por la Consejera del pueblo Kawésqar en el caso del proyecto "Piscicultura de Re-circulación Lago Balmaceda", la Corte Suprema descartó la interpretación formalista del principio de congruencia que utilizó el Tercer Tribunal Ambiental para omitir pronunciarse sobre la ausencia de las reuniones previas reguladas en el artículo 86 del Reglamento del SEIA, toda vez que:

"[...] tal norma se vincula con aquellas cuestiones que fueron objeto de observaciones, vinculadas [...] a la falta de consulta indígena y participación de pueblos originarios a pesar de que en el área de influencia del proyecto existen tierras indígenas en las que se realizan actividades por parte de una comunidad que tiene tal carácter" 59 .

Si bien el SEA reconoció expresamente que no efectuó las reuniones del artículo 86, quiso justificar dicha omisión apoyándose en las reuniones realizadas en el marco de las actividades de participación ciudadana y en un análisis unilateral de la información que había

\footnotetext{
52 Tercer Tribunal Ambiental, Rol № R-78-2018, de 7 de junio de 2019, considerando 67ㅇ.

53 Tercer Tribunal Ambiental, Rol № R-9-2019, de 27 de noviembre de 2019.

54 Tercer Tribunal Ambiental, Rol № R-12-19 (acumulado al Rol № R-14-2019 y R-15-2019), de 31 de marzo de 2020.

55 Tercer Tribunal Ambiental, Rol № R-14-2020, de 22 de septiembre de 2020.

${ }^{56}$ Tercer Tribunal Ambiental, Rol № R-13-2020, de 23 de octubre de 2020.

${ }^{57}$ En este sentido, ver: Tercer Tribunal Ambiental, Rol № R-9-2019, de 27 de noviembre de 2019, considerandos 15으 20으.

${ }^{58}$ En idénticos términos: Tercer Tribunal Ambiental, Rol № R-14-2020, de 22 de septiembre de 2020, considerando 67우 y Tercer Tribunal Ambiental, Rol № R-13-2020, de 23 de octubre de 2020, considerando 73ㅇ. Con todo, en una sentencia más reciente relativa al proyecto "Centro de Cultivo de Salmónidos Seno Taraba", el Tercer Tribunal Ambiental parece volver a reconocer la importancia de las reuniones del art. 86 del Reglamento del SEIA, no obstante estimó que en el caso concreto no correspondía llevarlas a cabo por no acreditarse la cercanía del proyecto a los grupos humanos indígenas. Ver: Tercer Tribunal Ambiental, Rol № 20-2019, de 27 de enero de 2021, considerando 106․ La sentencia tiene un voto de prevención en este punto que reconoce de manera intensa la función epistémica de las referidas reuniones, pues a través de ellas: "[...] pudo haberse obtenido información valiosa para el proceso de evaluación, que los mismos grupos humanos hubieran proporcionado, y que hubieran permitido, ya con mayor tranquilidad, presumiblemente haber descartado los efectos adversos del art. 11 letras c), d), e), o f) de la Ley 19.300 o confirmado tal susceptibilidad de afectación" (considerando 4ㅇ del voto preventivo del Ministro Retamal).

${ }^{59}$ Corte Suprema, Rol № 36.919-2019, de 22 de febrero de 2021, considerando 12 ․
} 
aportado el titular durante la evaluación ambiental del proyecto. Al rechazar estas alegaciones, la Corte Suprema sostuvo que estas reuniones:

"[...] tiene por objetivo no solo la aplicación del artículo 48 del Reglamento, sino que nutrir al ente que debe evaluar el proyecto, de información necesaria [para] el correcto desempeño de sus funciones, cuestión que se impone por el respeto irrestricto del principio preventivo y que, además, está previsto, como una forma de cumplimiento de los compromisos que surgen de la suscripción del Convenio 169 de la OIT.

$[\ldots]$

El SEA reconoce que no efectuó las reuniones del artículo 86 [...]; sin embargo, pretende soslayar su omisión esgrimiendo la realización de reuniones en el marco de las actividades de participación ciudadana, cuestión inadmisible, toda vez que las reuniones debe realizarse con el objetivo especifico de protección de los pueblos originarios que pudieren verse afectados por la ejecución del proyecto a evaluar. Es así como los pueblos originarios que pudieran ser afectados por un proyecto, a través de este mecanismo[] entregan información relevante acerca de aprehensiones y cuestionamientos que les surjan vinculados a la realización de las obras y la forma en que ellas influirán en sus sistemas de vida, la exposición que permite determinar la forma específica en que el proyecto les perturba, cuestión relevante si se considera que, en el caso de la Declaración de Impacto Ambiental, no existe la obligatoriedad de una consulta indígena, en la medida que el titular del proyecto demuestre de forma exhaustiva que no se dan los efectos del artículo 11 letras c) y d) de la Ley $N^{\circ} 19.300^{\prime \prime} 60$.

De esta manera, a juicio de la Corte, cuando lo que se está investigando es la afectación de grupos indígenas que mantienen sistemas de vida, formas de conocimiento y cosmovisiones propias, no se puede prescindir de la forma más fiable de recolección de datos: el dialogo intercultural directo con ellas, por intermedio de sus propias organizaciones e instituciones. Esto dado que, en el ámbito sociocultural, ni el Estado ni los titulares podrán comprender a cabalidad los efectos positivos o negativos que un determinado proyecto podría tener para dichos grupos, quienes son los principales expertos en lo que a sus sistemas de vida, culturas y territorio se refiere.

Aun cuando los criterios articulados por el Tercer Tribunal Ambiental en el caso del proyecto "Ampliación Minicentral Hidroeléctrica Las Flores”, así como por la Corte Suprema en el caso de la "Piscicultura de Re-circulación Lago Balmaceda", representan un avance hacia una comprensión epistémica de la participación de los pueblos indígena en la etapa de la determinación de la existencia (o no) de su SAD en relación a un proyecto sometido a evaluación ambiental, ambos tribunales persisten en el problema de exigir una afectación de magnitud en esta fase. Esto implica confundir el elemento gatillante de la consulta indígena (la SAD) con su finalidad (indagar de qué manera una determinada medida afecta a un grupo indígena), truncando la función epistémica de este instrumento para la formación de la evidencia en el contexto de los procedimientos de evaluación ambiental.

No ocurre lo mismo con la reciente sentencia del Primer Tribunal Ambiental en el caso del proyecto "Prospección Minera Norte Abierto sector Caspiche", que acogió la reclamación interpuesta por la Comunidad Colla de Río Jorquera y sus Afluentes, ordenando que el proyecto, evaluado por DIA, reingresara a al SEIA bajo la modalidad de un EIA con el correspondiente proceso de consulta indígena. Para arribar a dicha decisión, el Tribunal abandona el criterio de significancia del impacto como requisito de procedencia de la consulta, pues ella:

“[...] no puede ser el único criterio que guíe la protección de poblaciones o pueblos protegidos ya que precisamente es esa protección específica la que debe motivar cualquier tipo de decisión que los pueda afectar.

$[\ldots]$

${ }^{60}$ Corte Suprema, Rol № 36.919-2019, de 22 de febrero de 2021, considerando 12ㅇ, considerando 15ㅇ․ 
Que, cabe señalar que el concepto de significancia que pretende ser aplicado por la recurrida es un elemento que debe ser analizado a la luz de la autodeterminación cultural que rige en cada pueblo indígena. No es posible imponer o desconocer los propios criterios de poblaciones con cosmovisiones distintas, esto es, la visión de sus propias prioridades, y que la misma normativa reconoce en su afán de protección $[\ldots]^{61}$.

Como es posible apreciar, el Primer Tribunal Ambiental desestima las alegaciones del SEA referidas a la necesidad de un impacto de magnitud sobre un pueblo indígena, toda vez que ello invisibiliza los conocimientos de quienes, en definitiva, están en mejor posición de indicar cómo y en qué medida sus intereses serán afectados. El razonamiento expuesto por el Tribunal encuentra asidero en nuestra normativa ambiental, toda vez que ella:

"[...] no puede alentar una concepción restrictiva de protección a uno de sus elementos más
sensibles como es el medio humano, máxime cuando de su interpretación no es posible advertir
tal dirección. Por el contrario, es el mismo artículo 11 literal d) de la Ley № 19.300 el que discurre
sobre la hipótesis de la susceptibilidad de afectación y no de impactos significativos, como pudiese
entenderse de los otros literales. Que, es sólo de esta manera que puede entenderse
armónicamente el alcance y contenido del Convenio № 169 y el derecho ambiental interno" 62 .

El Tribunal, en lugar de negar la autonomía del artículo 11 d) de la Ley № 19.300, subordinando su aplicación a la concurrencia de otros "impactos significativos", como había ocurrido hasta entonces en la jurisprudencia, resalta la importancia de la SAD como criterio modulador e interpretativo de los demás literales y del reglamento, pues:

“[...] si bien los artículos 70, 8 y 10 del RSEIA originalmente se han previsto como complemento o detalle de los literales c), d) y f) del artículo 11 de la Ley № 19.300, respectivamente, la remisión que hace el artículo 85 del mismo Reglamento debe aplicarse teniendo como piedra angular la susceptibilidad de afectación a la que se refiere el literal d) del artículo $11^{\prime \prime} 63$.

Esta sentencia representa un hito importante en la protección de los derechos de los pueblos indígenas en la judicatura ambiental especializada. En efecto, no sólo se trata de la primera vez que un tribunal ambiental ordena un proceso de consulta indígena, sino que, además, interpreta nuestro ordenamiento jurídico ambiental a la luz de los estándares del Convenio 169, reconociendo la SAD como elemento gatillante del deber de consulta a partir de una lectura, a nuestro juicio, correcta del artículo 11 letra d) de la Ley № 19.300. Bajo esta interpretación, tal como lo habíamos adelantado, la homologación de los "impactos significativos" con la SAD deviene en un problema más aparente que real, y la consecuencia que se impone es que los proyectos sometidos al SEIA que generan SAD deberán ingresar por un EIA, por concurrir el impacto ambiental regulado en el artículo 11 letra d) de la Ley № 19.300 .

\subsection{La dimensión epistémica de la consulta previa en la jurisprudencia relativa al Reglamento general de consulta}

Un desarrollo interesante que reconoce la función epistémica de la consulta previa se puede observar en la jurisprudencia de protección reciente en el contexto de la aplicación del Reglamento general de consulta. Si bien los tribunales superiores de justicia al conocer de impugnaciones de medidas inconsultas efectuadas por comunidades indígenas a través de recurso de protección, han exigido un alto grado de certeza y magnitud (significancia) a la

\footnotetext{
61 Primer Tribunal Ambiental, Rol № R-38-2020, de 21 de abril de 2021.

62 Primer Tribunal Ambiental, Rol № R-38-2020, de 21 de abril de 2021.

63 Primer Tribunal Ambiental, Rol № R-38-2020, de 21 de abril de 2021.
} 
afectación requerida para gatillar en un proceso de consulta previa, esta actitud deferente hacia los estándares del Reglamento general de consulta podría estar cambiando.

Esto se aprecia con claridad en la sentencia de la Corte Suprema que acogió dos recursos de protección interpuestos por diversas personas naturales y organizaciones mapuche, en contra de dos actos administrativos dictados por la Corporación Nacional Forestal (CONAF) y el Gobierno Regional de la Araucanía, respectivamente, que buscaban darle continuidad al proyecto "Mejoramiento Integral Zona de Uso Público Parque Nacional Villarrica". Esta última, correspondía a una iniciativa de inversión pública que por sus características y dimensiones no debía hacer ingreso obligatorio al SEIA. Al resolver los recursos, el Máximo Tribunal revocó las sentencias de la Corte de Apelaciones de Temuco que, a su vez, habían rechazado las acciones de los recurrentes por considerar que fueron interpuestas extemporáneamente y no habían acreditado de qué forma los actos impugnados los iban a afectar, cuestión que además estaba respaldada por el informe evacuado por la Subsecretaría de Servicios Sociales en el marco del artículo 13 del Reglamento general de consulta ${ }^{64}$.

Tras rechazar los argumentos que decían relación con la supuesta extemporaneidad de los recursos de protección, la Corte señaló:

\begin{abstract}
"Que la participación de los pueblos afectados por un proyecto en el PCl, les permite ser parte de un intercambio de información relevante acerca de las obras a realizar y la forma en que ellas influirán en sus sistemas de vida, la exposición de los puntos de vista de cada uno de los potenciales afectados de manera de determinar la forma específica en que el proyecto les perturba, el ofrecimiento de medidas de mitigación, compensación y/o reparación y, finalmente, la formalización de acuerdos en un plano de igualdad, otorgando a los pueblos indígenas la posibilidad de influir de manera real y efectiva en las decisiones públicas que sean de su interés" ${ }^{\prime 65}$. A partir de lo anterior, la Corte señaló que:

"[...] a la luz de lo razonado, no resulta admisible el argumento de la autoridad administrativa, en orden a que la consulta indígena no procedía por no existir afectación a las comunidades y personas naturales en nombre de quienes se recurre, puesto que la obligatoriedad de este proceso exige únicamente una afectación potencial, cuya materialización será precisamente analizada en el marco de la señalada consulta.

Por tanto, para que exista susceptibilidad de afectación directa en los términos exigidos por el Convenio № 169 de la OIT, es necesario que se encuentre establecido en autos que se verifica dicha potencialidad, cuestión que en estos antecedentes efectivamente ocurre, por cuanto se trata [...] de la construcción de baños, duchas, casetas, sitios de camping, terrazas, estacionamientos, alcantarillado y agua potable, sistemas de solución eléctrica, remodelaciones, gaviones, tranqueras, letreros, señales y otras obras complementarias, en cuatro sectores del parque, terrenos dentro de los cuales, si bien no se hallan emplazadas comunidades indígenas, existen espacios donde personas pertenecientes a pueblos originarios realizan prácticas religiosas y culturales, lo cual es reconocido por Conaf" 66 .
\end{abstract}

Este razonamiento, que ya había sido expresado hasta este momento en votos de minoría ${ }^{67}$, representa un avance importante en la jurisprudencia de la Corte Suprema, pues por una parte interpreta conforme al Convenio 169 los exigentes requisitos del Reglamento para dar curso a un proceso de consulta, y por otro, reconoce la función epistémica de la consulta, pues precisamente a través de ella se determinará, a partir de la información

\footnotetext{
${ }^{64}$ Corte de Apelaciones de Temuco, Rol № 17311-2019, de 26 de octubre de 2020; Corte de Apelaciones de Temuco, Rol № 62862019, de 26 de octubre de 2020.

65 Corte Suprema, Rol №138.439-2020 (acumulado al Rol № 140.342-2020), de 29 de marzo de 2021, considerando 8ㅇ.

${ }^{66}$ Corte Suprema, Rol № 138.439-2020 (acumulado al Rol № 140.342-2020), de 29 de marzo de 2021, considerando 10 ㅇ.

${ }^{67}$ Ver: Corte Suprema, Rol № 20.913-2019, de 1 de octubre de 2019, considerando 3o del voto disidente de los Ministros Sergio Muñoz y Ángela Vivanco: “[...] no resulta admisible el argumento de la recurrida, reproducido también por la Seremi de Vivienda y Urbanismo, en orden a que la consulta indígena no resultaba procedente por no encontrarse acreditada la afectación a las comunidades y personas naturales recurrentes, puesto que la procedencia de este proceso exige únicamente una afectación potencial, en tanto su efectividad será precisamente analizada en el marco de la señalada consulta". En similares términos: Corte Suprema, Rol № 2.025-2019, de 6 de enero de 2020, considerando 2o del voto disidente del Ministro Sergio Muñoz.
} 
entregada por los pueblos indígenas en un contexto de diálogo intercultural, la forma en que sus intereses se verán efectivamente afectados por la decisión a adoptar.

Ahora bien, esta interpretación de la Corte Suprema en el conocimiento de recursos de protección sobre casos gobernados por el Reglamento general de consulta contrasta con la interpretación que hasta el momento ha dominado la jurisprudencia de los Tribunales Ambientales respecto al Reglamento del SEIA y la Ley $N^{\circ} 19.300$. Así, mientras que en sede de protección se exige sólo SAD para dar curso a un proceso de consulta -pese a que el reglamento exige injustificadamente un "impacto significativo y específico"-, en sede ambiental se requiere una "evidencia de afectación directa y significativa" ${ }^{68}$, en circunstancias de que esta exigencia no se desprende del tenor del literal d) del artículo 11 La ley № 19.300. Esto se ha traducido en que la función epistémica de los mecanismos de diálogo intercultural en el marco del SEIA haya quedado relegada a las reuniones previas del art. 86 del reglamento del SEIA, mas no a la consulta indígena. Lo anterior, es sin perjuicio de la sentencia del Primer Tribunal Ambiental en el caso del proyecto "Prospección Minera Norte Abierto sector Caspiche", que, de confirmarse por la Corte Suprema, podría marcar un antes y un después en lo que dice relación con el ensamblaje regulatorio restrictivo de la SAD en el SEIA.

\section{Conclusiones}

En este trabajo hemos argumentado que a nivel internacional los derechos de participación de los pueblos indígenas constituyen mecanismos fundamentales a través de los cuales estos grupos pueden participar en la formación de la prueba y la evidencia durante los procesos de toma de decisiones estatales. Si bien, tras la ratificación del Convenio 169, Chile incorporó a su ordenamiento interno disposiciones específicas sobre la consulta previa, se ha instalado en este país una concepción puramente procedimental de este derecho, dando lugar a un modelo regulatorio restrictivo, en el que la afectación exigida para gatillar la consulta (la SAD) debe ser de magnitud (significativa) y cierta (altamente probable), y donde el control de su determinación en un caso concreto permanece férreamente controlada por los órganos estatales responsables y los titulares de proyectos (en el caso del SEIA).

Lo anterior, ha implicado alterar el principio que a este respecto opera a nivel internacional, al trasladar la discusión sobre la efectividad de la ocurrencia (o no) de una afectación, lo que debiera ser el resultado de la consulta, al momento de la evaluación en concreto de su procedencia. Esta forma de interpretar los presupuestos de la consulta en el SEIA, junto con distorsionar el estándar internacional de afectación exigido para dar curso a una consulta, impide que este derecho pueda cumplir con la doble función epistémica para la cual ha sido diseñada: habilitar la participación colectiva de los pueblos indígenas como agentes de conocimientos propios y validos, y operar como un mecanismo de visibilización de impactos para la protección de los derechos indígenas en contextos de diversidad cultural.

Sin perjuicio de esto, es posible observar algunos avances jurisprudenciales en que, pese a las limitaciones impuestas por el modelo regulatorio de la consulta previa indígena en Chile, han comenzado ha desarrollar interpretaciones conforme al Convenio 169 y a enfatizar la función epistémica de este derecho de los pueblos indígenas en el contexto de los procesos de toma de decisiones. Así se observa en los criterios articulados por el Tercer Tribunal Ambiental en el caso del proyecto "Ampliación Minicentral Hidroeléctrica Las Flores", así como por la Corte Suprema en el caso de la "Piscicultura de Re-circulación Lago Balmaceda", los cuales representan un avance hacia una comprensión de la función epistémica de la participación de los pueblos indígena en la etapa de la determinación de la existencia (o no) de su SAD en relación a un proyecto sometido a evaluación ambiental. Sin embargo, como se señaló, esta jurisprudencia persististe en el problema de trasladar la discusión respecto al alcance y magnitud de la afectación al momento de la evaluación en concreto de la procedencia de la

${ }^{68}$ Tercer Tribunal Ambiental, Rol № R-38-2016, de 28 de diciembre de 2016, considerando 37ㅇ․ 
consulta, confundiendo el elemento gatillante de la consulta indígena con su finalidad: indagar de qué manera una determinada medida afecta a un grupo indígena. Esta cuestión resulta compleja, toda vez que al descartar la procedencia de la consulta por no existir una afectación directa acreditable de ante mano, se prescinde de información relevante y de los conocimientos indígenas que son claves para la preservación de la biodiversidad.

Sin perjuicio de lo anterior, este entendimiento restrictivo de la SAD en el contexto del SEIA pareciera estar entrando en cuestionamientos. De esto da cuenta la reciente decisión del Primer Tribunal Ambiental en el caso del proyecto "Prospección Minera Norte Abierto sector Caspiche". Esta sentencia representa un hito importante en la protección de los derechos de los pueblos indígenas en la judicatura ambiental, ya que no sólo se trata de la primera vez que un tribunal ambiental ordena un proceso de consulta indígena, sino que además, interpreta nuestro ordenamiento jurídico ambiental a la luz de los estándares del Convenio 169, reconociendo la SAD como elemento gatillante del deber de consulta a partir de una correcta lectura del artículo 11 letra d) de la Ley № 19.300. Esto, tal como lo señalamos, convierte la homologación de los "impactos significativos" con la SAD en un problema más aparente que real.

Por otra parte, en el ámbito de casos gobernados por el reglamento general de consulta, se destaca la sentencia de la Corte Suprema en el caso proyecto "Mejoramiento Integral Zona de Uso Público Parque Nacional Villarrica". En este caso, el Máximo Tribunal reconoce que el elemento desencadenante de un proceso de consulta es únicamente la constatación preliminar de una afectación potencial, toda vez que la efectividad de la ocurrencia (o no) de dicha afectación y su alcance, solo podrá ser analizada en el marco de la consulta. Desde esta perspectiva, la consulta previa indígena cumple una función epistémica clave en los procesos de toma de decisiones, al apoyar la generación de evidencia respecto a los impactos que una determinada medida puede tener sobre los pueblos indígenas, con el objetivo de que la decisión final que se adopte se acomode a las perspectivas, intereses y derechos sustantivos de estos grupos, históricamente marginados de las instancias decisorias al interior de los Estados de colonos que los han subordinado.

\section{BIBLIOGRAFÍA CITADA}

ANAYA, JAMES (1991): "Indigenous Rights Norms in Contemporary International Law", en: Arizona Journal of International and Comparative Law (Vol. 8, № 2), pp. 1-40.

ANAYA, JAMES (2000): Indigenous Peoples in International Law (Nueva York, Oxford University Press).

AnAYA, JAmes (2012): "Comentarios del Relator Especial sobre los derechos de los pueblos indígenas en relación con el documento titulado: 'Propuesta de gobierno para nueva normativa de consulta y participación indígena de conformidad a los artículos 60 y 70 del Convenio № 169 de la Organización Internacional del Trabajo', Chile, Noviembre de 2012". Disponible en: http://unsr.jamesanaya.org/docs/special/2012-11-29-unsr-comentarios-apropuesta-reglamento-consulta-chile.pdf [visitado el 08 de mayo de 2021].

Aninat, Isabel (2014): "Los desafíos pendientes del Convenio 169", en: Punto de Referencia, Centro de Estudios Públicos (№ 369), pp. 1-11.

AylWin, JosÉ; Meza-LopehandíA, Matías y Yáñez, Nancy (2013): Los pueblos indígenas y el derecho (Santiago de Chile, LOM ediciones).

CARMONA, CRISTÓBAL (2020): “¿La identidad cultural como límite de la Consulta Indígena en el SEIA? Notas para una subversión de la comprensión 'procedimental' de la consulta", en: Faundes, Juan y Ramírez, Silvina (Eds.)., Derecho fundamental a la identidad cultural. Abordajes plurales desde América Latina (Santiago, RIL editores-Universidad Autónoma de Chile), pp. 343-359. 
COMISIÓN DE EXPERTOS en APLICACIÓN de CONVENIOS y RECOMENDACIONES (2006): "Observación (CEACR) Convenio sobre pueblos indígenas y tribales, 1989 (núm. 169) - Guatemala (Ratificación: 1996), Adopción: 2005, Publicación: 95a reunión CIT". Disponible en: https://www.ilo.org/dyn/normlex/es/f?p=NORMLEXPUB:13100:0::NO::P13100_COMMENT_ID :2261253 [visitado el 07 de julio de 2021].

COMISIÓN DE EXPERTOS EN APLICACIÓN dE CONVENIOS Y RECOMENDACIONES (2009): "Observación General sobre el Convenio núm. 169, 79a reunión, 2008". Disponible en: https://www.ilo.org/dyn/normlex/es/f?p=NORMLEXPUB:13100:0::NO::P13100_COMMENT_ID ,P11110_COUNTRY_ID,P11110_COUNTRY_NAME,P11110_COMMENT_YEAR:3066698,,,2008 [visitado el 8 de julio de 2021].

COMISIÓN DE EXPERTOS en APLICACIÓN de CONVENIOS y ReCOMENDACIONES (2011): "Observación 2010/81. Pueblos indígenas y tribales (CEACR-2010-O-S20-GEN-Sp.doc/v.3)". Disponible en: https://www.ilo.org/wcmsp5/groups/public/---ed_norm/---

normes/documents/meetingdocument/wcms_305844.pdf [visitado el 08 de mayo de 2021].

Comisión InTERAmericana de DeRechos Humanos (2009): "Derechos de los pueblos indígenas y tribales sobre sus tierras ancestrales y recursos naturales: normas y jurisprudencia del sistema interamericano de derechos humanos (OEA/Ser.L/V/II. Doc. 47/15)". Disponible en: http://cidh.org/countryrep/tierrasindigenas2009/Tierras-Ancestrales.ESP.pdf [visitado el 08 de mayo de 2021].

Comisión INTERAmericana de Derechos Humanos (2015): "Pueblos indígenas, comunidades afrodescendientes y recursos naturales: protección de derechos humanos en el contexto de actividades de extracción, explotación y desarrollo (OEA/Ser.L/V/II. Doc. 47/15)". Disponible en: http://www.oas.org/es/cidh/informes/pdfs/industriasextractivas2016.pdf [visitado el 08 de mayo de 2021].

Consejo de DeRechos Humanos de las Naciones Unidas (2009): “Promoción y protección de todos los derechos humanos, civiles, políticos, económicos, sociales y culturales, incluido el derecho al desarrollo. Informe del Relator Especial sobre la situación de los derechos humanos y las libertades fundamentales de los indígenas, James Anaya (A/HRC/12/34, Original: inglés)". Disponible en: https://www.acnur.org/fileadmin/Documentos/BDL/2010/8057.pdf [visitado el 08 de mayo de 2021].

CORDERO, LUIS (2013): "Derecho administrativo y Convenio 169: La procedimentalización de los conflictos como consecuencia de soluciones incompletas", en: Olea, Helena (Ed.)., Derecho y Pueblo Mapuche. Aportes para la discusión (Santiago, Universidad Diego Portales), pp. 69-85.

DonOSO, SEBASTIÁN (2012): “La participación ciudadana del SEIA como consulta indígena: Alcances a la jurisprudencia de la Corte Suprema y en particular a un reiterado voto de minoría", en: Libertad y Desarrollo, Anuario de doctrina y jurisprudencia. Sentencias destacadas 2011. Una mirada desde la perspectiva de las políticas pública (Santiago, Ediciones LYD/Libertad y Desarrollo), pp. 173-204.

DoNOSO, SEBASTIÁN (2014): “Declaraciones de impacto ambiental y consulta indígena en el SEIA: Comentario a la tendencia de homologación de impactos ambientales que exigen un EIA y a susceptibilidad de afectación directa del Convenio 169 OIT", en: Libertad y Desarrollo, Anuario de doctrina y jurisprudencia. Sentencias destacadas 2013. Una mirada desde la perspectiva de las políticas públicas (Santiago, Ediciones LYD/Libertad y Desarrollo), pp. 57-83.

EIDE, ASBJ ØRN (2010): “Los pueblos indígenas, el Grupo de Trabajo sobre Poblaciones Indígenas y la adopción de la Declaración de la ONU sobre los Derechos de los Pueblos Indígenas", en: Charters, Calire y Stavenhagen, Rodolfo (Ed.)., El Desafío de la Declaración. Historia y Futuro de la Declaración de la ONU sobre Pueblos Indígenas (Copenhague, IWGIA), pp. 34-49. 
FRICKER, MIRANDA (2007): Injusticia epistémica. El poder y la ética del conocimiento (Barcelona, Herder).

Fundación PARA el Debido PROCESo (2015): Derecho a la consulta y al consentimiento previo, libre e informado en América Latina. Avances y desafíos para su implementación en Bolivia, Brasil, Chile, Colombia, Guatemala y Perú (Washington DC, Fundación para el Debido Proceso).

GoBIERNO DE CHILE (2012): "Informe final. Proceso de consulta indígena sobre el reglamento del SEIA, guías de procedimientos de participación ciudadana y de apoyo para la evaluación de alteraciones significativas sobre pueblos originarios". Disponible en: https://sea.gob.cl/sites/default/files/migration_files/archivos/contenidos/02_Informe_Final_C onsulta_Indigena_RSEIA_SEA.pdf [visitado el 08 de mayo de 2021].

GuerRA, FeliPe (2017): “Los Tribunales Ambientales en la implementación de los derechos indígenas durante la evaluación ambiental de proyectos de inversión en Chile", en: Revista Justicia Ambiental (№ 9), pp. 19-38

INSTITUto NACIONAL DE DeRECHOS Humanos (2013): "El deber de consulta previa en la Propuesta de reglamento del Sistema de Evaluación Ambiental". Disponible en: https://bibliotecadigital.indh.cl/bitstream/handle/123456789/529/minuta?sequence $=1$

[visitado el 08 de mayo de 2021].

Kelly, SARAH (2019): "Megawatts mask impacts: Small hydropower and knowledge politics in the Puelwillimapu, Southern Chile", en: Energy Research \& Social Science (Vol. 54), pp. 224235.

LARSEN, Peter (2019): “Contextualising ratification and implementation: a critical appraisal of ILO Convention 169 from a social justice perspective", en: The International Journal of Human Rights (Vol. 24, № 2-3), pp. 94-111.

Larsen, Peter (2016): “The 'New Jungle Law': Development, Indigenous Rights and ILO Convention 169 in Latin America", en: International Development Policy (Vol. 7, № 1), pp. 119.

LÓPEZ, RICARDO y MOHR, TANIA (2014): "Susceptibilidad de afectación directa en la consulta previa del Convenio 169. Análisis de Normas Previstas y de su trato en la Jurisprudencia. ¿Una cuestión de derecho?", en: Revista de Derecho (Valdivia) (Vol. 27, № 1), pp. 105-126.

MezA-Lopehandía, Matías (2018): “Frustred Multiculturalism. (Neo)Liberalism and the Mapuche People", en: Marshall, Pablo (Ed.), Citizenship and Disadvantaged Groups in Chile (USA, Lexington Books), pp. 123-139.

MEZA-Lopehandía, MATías (2016): “La jurisprudencia del multiculturalismo en Chile: La consulta previa ante tribunales", en: Revista de Ciencias Sociales (№ 69), pp. 13-52.

Millaleo, Salvador (2014): “¿Gobernar Consultado? Análisis comparativo respecto a los problemas en relación a las bases y objetos de la consulta indígena (Convenio 169) de los reglamentos aprobados en Chile", en: Cariman, Braulio; Collipal, María; Huiechaqueo, María; Licanqueo, Elizabeth; Loncon, Elisa; Loncon, Lautaro; Millaleo, Salvador; Millapan, Diva; Namuncura, Domingo; Noriega, Leyla; Valdés, Marcos, ¿Chile indígena? Desafíos y oportunidades para un nuevo trato (Santiago, Fundación Chile), pp. 52-102.

NIEZEN, RONALD (2003): The Origins of Indigenism. Human rights and the politics of identity (Berkley, London, University of California Press).

NúÑEZ, MANUEL (2015): “Estándares de cautela, consulta indígena y fundamentación en los procesos de evaluación de impacto ambiental. Comentarios a El Morro", en: Libertad y Desarrollo., Sentencias Destacadas 2014 (Santiago, Ediciones LYD/Libertad y Desarrollo), pp. 19-36. 
ObSERVATORIO CIUDADANo (2018): "El Convenio 169 de la OIT sobre pueblos indígenas y tribales a 10 años de su ratificación por el Estado de Chile: Análisis crítico de su cumplimiento (Chile, Observatorio Ciudadano-Central Unitaria de Trabajadores)". Disponible en: https://cut.cl/cutchile/wp-content/uploads/2019/01/Informe-OC-CUT-a-10-an\%CC\%83os-deConvenio169-baja.pdf [visitado el 8 de julio de 2021].

Oficina Internacional del Trabajo (2019): Aplicando el Convenio sobre Pueblos Indígenas y Tribales, 1989 (Núm. 169): Extractos de informes y comentarios de los Órganos de Control de la OIT (Guatemala, Departamento de Normas Internacionales del Trabajo).

SCHMIDT, KATE C.S. (2019): “Epistemic Justice and Epistemic Participation. Dissertation presented to The Graduate School of Washington University in partial fulfillment of the requirements for the degree of Doctor of Philosophy". Disponible en: https://openscholarship.wustl.edu/cgi/viewcontent.cgi?article=2819\&context=art_sci_etds [visitado el 8 de julio de 2021].

Servicio de Evaluación Ambiental (2016): "Instructivo sobre implementación del proceso de consulta a pueblos indígenas en conformidad con el Convenio № 169 de la OIT en el Sistema de Evaluación de Impacto Ambiental, Of. Ord. D.E. № 161116". Disponible en: https://www.sea.gob.cl/sites/default/files/migration_files/instructivos/of._ord._ndeg_161116. pdf [visitado el 07 de julio de 2021].

\section{JURISPRUDENCIA CITADA}

REQUERIMIENTO FORMULADO POR DIVERSOS DIPUTADOS PARA QUE EL TRIBUNAL RESUELVA LA Constitucionalidad del CONVENIO № 169, SObRe Pueblos Indígenas y TRIBales en Países INDEPENDIENTES, ADOPTADO POR LA ORGANIZACIÓN INTERNACIONAL DEL TRABAJO, EL 27 DE JUNIO DE 1989, DE ACUeRdo AL ARTíCULO 82, N² 2, DE LA CONSTITUCIÓN PolítICA DE LA RepúbliCA (2000): Tribunal Constitucional 04 de agosto de 2000 (requerimiento de inconstitucionalidad), en: https://www.tribunalconstitucional.cl.

Luis Alberto Araneda Necuman con Comité de Ministros (2016): Tercer Tribunal Ambiental 28 de diciembre de 2016 (reclamación), en: https://3ta.cl/fallos.

COMUNIDAd INDÍGENA SATURNINo LEAL NEIMAN Y OtROS CON COMISIÓN DE EVALUACIÓN AMBIENTAL de LoS Ríos (2018): Tercer Tribunal Ambiental 07 de junio de 2019 (reclamación), en: https://3ta.cl/fallos.

NAVARRO CON PINTO (2019): Corte Suprema 01 de octubre de 2019 (apelación protección), en: www.pjud.cl.

Marcela Caro loncuante con Director Ejecutivo de Servicio de EValuación Ambiental (2019): Tercer Tribunal Ambiental 27 de noviembre de 2019 (reclamación), en: https://3ta.cl/fallos.

Compañia Minera Nevada SPA con Servicio De Evaluacion Ambiental (2020): Corte Suprema 06 de enero de 2020 (casación en la forma y en el fondo), en: www.pjud.cl.

Comunidad Indígena El Manzano y otros con Servicio de EValuación Ambiental (2020): Tercer Tribunal Ambiental 31 de marzo de 2020 (reclamación), en: https://3ta.cl/fallos.

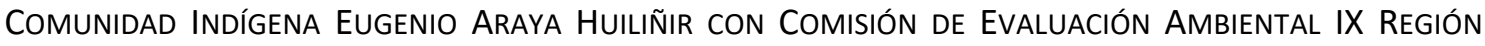
(2020): Tercer Tribunal Ambiental 22 de septiembre de 2020 (reclamación), en: https://3ta.cl/fallos.

Comunidad Indígena Eugenio ARAya huiliñIR con Comisión de EValuación Ambiental IX Región (2020): Tercer Tribunal Ambiental 23 de octubre de 2020 (reclamación), en: https://3ta.cl/fallos. 
CANIUMÁn CON CoRporacion NACIONAL Forestal (2020): Corte de Apelaciones de Temuco 26 de octubre de 2020 (acción de protección), en: www.pjud.cl.

Caniumán con Gobierno Regional de la Araucanía (2020): Corte de Apelaciones de Temuco 26 de octubre de 2020 (acción de protección), en: www.pjud.cl.

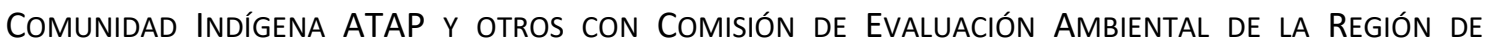
MAgALLANES (2021): Tercer Tribunal Ambiental 27 de enero de 2021 (reclamación), en: https://3ta.cl/fallos.

Marcela Caro loncuante con director Ejecutivo de Servicio de EValuación ambiental (2021): Corte Suprema 22 de febrero de 2021 (casación en el fondo), www.pjud.cl.

CANIUMÁN CON CORPORACION NACIONAL ForeStal (2021): Corte Suprema 29 de marzo de 2021 (apelación protección), www.pjud.cl.

Comunidad Indígena Colla Río JoRquera y sus afLUentes CON SEA (2021): Primer Tribunal Ambiental 21 de abril de 2021 (reclamación), https://www.1ta.cl/sentencias.

\section{NORMAS JURÍDICAS CITADAS}

LEY № 19.300, sobre Bases Generales del Medio Ambiente. Diario oficial, 09 de marzo de 1994.

LEY № 20.600, que Crea los Tribunales Ambientales. Diario oficial, 28 de junio de 2012.

CONVENIO № 169 Sobre Pueblos Indígenas y Tribales en Países Independientes de la Organización Internacional del Trabajo. 27 de junio de 1989.

DeCRETO SUPREMO № 40, Aprueba Reglamento del Sistema de Evaluación de Impacto Ambiental. Diario oficial, 12 de agosto de 2013.

DeCRETO SUPREMO № 66, Aprueba Reglamento que regula el procedimiento de consulta indígena en virtud del artículo 6 № 1 letra a) y № 2 del Convenio № 169 de la Organización Internacional del Trabajo y deroga normativa que indica. Diario oficial, 04 de marzo de 2014. 